\title{
Investigation of the Purcell effect in photonic crystal cavities with a 3D Finite Element Maxwell Solver
}

\section{Conference Paper}

Author(s):

Roemer, Friedhard; Witzigmann, Bernd; Chinellato, Oscar; Arbenz, Peter

Publication date:

2007-03

Permanent link:

https://doi.org/10.3929/ethz-b-000007906

Rights / license:

In Copyright - Non-Commercial Use Permitted

Originally published in:

Optical and Quantum Electronics 39(4-6), https://doi.org/10.1007/s11082-007-9089-1 


\title{
Investigation of the Purcell effect in photonic crystal cavities with a 3D Finite Element Maxwell Solver
}

\author{
Friedhard Römer • Bernd Witzigmann • \\ Oscar Chinellato • Peter Arbenz
}

Received: 20 September 2006 / Accepted: 19 January 2007 / Published online: 8 June 2007

C Springer Science+Business Media, LLC 2007

\begin{abstract}
Photonic crystal cavities facilitate novel applications demanding the efficient emission of incoherent light. This unique property arises when combining a relatively high quality factor of the cavity modes with a tight spatial constriction of the modes. While spontaneous emission is desired in these applications the stimulated emission must be kept low. A measure for the spontaneous emission enhancement is the local density of optical states (LDOS). Due to the complicated three dimensional geometry of photonic crystal cavities the LDOS quantity has to be computed numerically. In this work, we present the computation of the LDOS by means of a 3D Finite Element (FE) Maxwell Solver. The solver applies a sophisticated symmetry handling to reduce the problem size and provides perfectly matched layers to simulate open boundaries. Different photonic crystal cavity designs have been investigated for their spontaneous emission enhancement by means of this FE solver. The simulation results have been compared to photoluminescence characterizations of fabricated cavities. The excellent agreement of simulations and characterizations results confirms the performance and the accuracy of the 3D FE Maxwell Solver.
\end{abstract}

Keywords Finite element method · Green's function · Microcavity · Photonic crystal . Purcell effect

\section{Introduction}

Spontaneous emission is not only an intrinsic property of the atoms or molecules in a specific material but is also governed by the electromagnetic properties of the host facilitating its supression as well as the enhancement. The latter effect has first been proposed by E.M. Purcell (Purcell et al. 1946) placing the emitter into an electromagnetic resonator tuned to the

F. Römer $(\varangle) \cdot$ B. Witzigmann

Integrated Systems Laboratory, ETH Zürich, Zürich 8092, Switzerland

e-mail: froemer@iis.ee.ethz.ch

O. Chinellato $\cdot$ P. Arbenz

Institute of Computational Science, ETH Zürich, Zürich 8092, Switzerland 


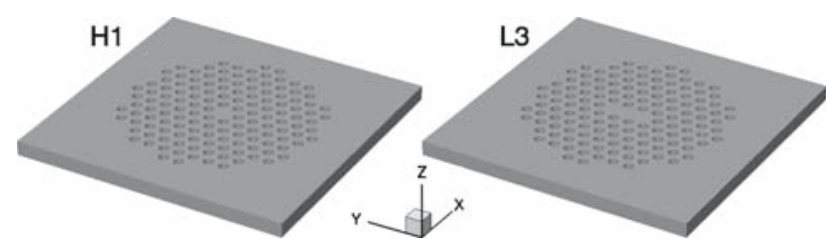

Fig. 1 Schematic of a H1 and a L3 photonic crystal cavity. The H1 cavity has got one defect hole, and the L3 cavity has got three defect holes in line

emission wavelength. The simplified analytic model derived for closed cavities indicates that the spontaneous emission enhancement factor $\beta$ is proportional to the quality factor $Q$ and inverse proportional to the volume $V$ (Meschede 1992) at resonance. Therefore this effect as well as its counterpart- the spontaneous emission suppression-can only be observed in optical cavities of about wavelength size.

Meanwhile, several novel applications of light emitters in the field of sensing, material science, fundamental research and illumination (Alivisatos 2004; Imamoglu 2003; Streubel et al. 2002) rely on the efficient generation of spontaneous emission with a coherence time corresponding to the cavity lifetime or the generation of single photons. While this type of emission is highly desired, stimulated emission enlarging the coherence time by orders of magnitude or leading to the emission of correlated photons has to be suppressed as far as possible. To this end, we consider the zero dimensional laser rate equation model (Coldren and Corzine 1995), according to which stimulated emission in a cavity becomes dominant when the photon generation rate in a particular cavity mode compensates for the cavity losses. The losses, in turn, are inverse proportional to the quality factor of the cavity. Hence, the quality factor governs the lasing threashold with respect to the cavity. The spontaneous emission efficiency does not only depend on a large quality factor but also on a small effective modal volume $V_{\text {eff }}$. This explains why microcavities can enhance the spontaneous emission while keeping the stimulated emission low. Nowadays, photonic crystal cavities yield quality factors up to $Q=10^{6}$ (Song et al. 2005) and such small field confinement as to render these cavities ideal for both lasing and stimulated emission. The design of photonic crystal cavities creates new challenges beyond the optimization of the quality factor.

As shown in Fig. 1 the photonic crystal cavities investigated in this work feature a periodic arrangement of holes in a thin slab of semiconductor material with some defect holes forming the cavity. Due to this geometry the paraxial wave approximation cannot be applied allowing only full 3D methods for simulation. A good candidate for the latter is the Finite Difference Time Domain (FDTD) method which is a well established tool for analyzing 3D photonic crystal cavity problems (Vučković etal. 2002). In this work we will present an analysis of photonic crystal cavities by means of a 3D Finite Element Method (FEM) Maxwell Solver, however. This solver is aimed at a fully coupled electro-opto-thermal simulation of semiconductor light emitters in the slowly varying amplitude regime (Witzigmann et al. 2000; Streiff et al. 2003). As the FEM solver facilitates an efficient iterative refinement of the eigenmodes of the optical cavity it provides better compatibility and much higher performance than a FDTD solver within this environment. Moreover, the FEM Maxwell solver provides the full dyadic Green's function $\mathcal{G}_{11}\left(\mathbf{r}, \mathbf{r}^{\prime}, k\right)$ in one computational step only as will be shown later.

The 3D FEM Maxwell solver presented in this work has been specifically designed for open cavities like photonic crystal cavities. It provides perfectly matched layers (PML) (Berenger 1995; Teixeira and Chew 2000) to simulate the open boundary condition and it exploits symmetries in order to reduce the size of the problem. The solver supports the solution 
of eigenproblems as well as source problems. The computation of the eigenmodes readily yields the quality factor of the cavity, one factor influencing the spontaneous emission. The effective volume being the other factor influencing spontaneous emission is not well defined in open cavities. Moreover, the solution provided by the aforementioned approximate model does not indicate any spatial dependence of the spontaneous emission enhancement. The closed model for spontaneous emission that holds in the weak coupling regime shows that spontaneous emission is proportional to the local density of optical states (LDOS) $\rho(\mathbf{r}, k)$ (Joulain et al. 2003; Henkel and Sandoghdar 1998). The LDOS in turn is closely related to the electromagnetic Green's functions of the cavity. One finds that (Joulain et al. 2003)

$$
\begin{aligned}
\rho(\mathbf{r}, k) & =\rho_{e}(\mathbf{r}, k)+\rho_{m}(\mathbf{r}, k) \\
& =\frac{k}{\pi c} \operatorname{Im}\left\{\operatorname{Tr}\left[\mathcal{G}_{11}(\mathbf{r}, \mathbf{r}, k)\right]\right\}+\frac{k}{\pi c} \operatorname{Im}\left\{\operatorname{Tr}\left[\mathcal{G}_{22}(\mathbf{r}, \mathbf{r}, k)\right]\right\}
\end{aligned}
$$

where $\mathcal{G}_{11}$ and $\mathcal{G}_{22}$ are the electric and the magnetic Green's functions evaluated at the position $\mathbf{r}$ for the wave number $k=\omega / c$, respectively. While Eq. (1) holds for the total density of states, the interband transition in quantum dots is only affected by the electric polarization and thus the electric Green's function $\mathcal{G}_{11}$ alone. We will therefore refer to the LDOS as the one related to the electric Green's function, $\rho_{e}(\mathbf{r}, k)$.

Hence, the ratio of the cavity LDOS to the free space LDOS yields the relative LDOS or the spontaneous emission enhancement factor $\beta(\mathbf{r}, k)=\rho_{e}(\mathbf{r}, k) / \rho_{e, v a c}(k)$. It is useful to resolve the factor $\beta(\mathbf{r}, k)$ for each principal axis yielding

$$
\begin{aligned}
& \beta_{x}(\mathbf{r}, k)=\frac{2 \pi}{k} \operatorname{Im}\left\{\hat{\mathbf{x}}^{T} \mathcal{G}_{11}(\mathbf{r}, \mathbf{r}, k) \hat{\mathbf{x}}\right\} \\
& \beta_{y}(\mathbf{r}, k)=\frac{2 \pi}{k} \operatorname{Im}\left\{\hat{\mathbf{y}}^{T} \mathcal{G}_{11}(\mathbf{r}, \mathbf{r}, k) \hat{\mathbf{y}}\right\} \\
& \beta_{z}(\mathbf{r}, k)=\frac{2 \pi}{k} \operatorname{Im}\left\{\hat{\mathbf{z}}^{T} \mathcal{G}_{11}(\mathbf{r}, \mathbf{r}, k) \hat{\mathbf{z}}\right\}
\end{aligned}
$$

where $\hat{\mathbf{x}}, \hat{\mathbf{y}}$, and $\hat{\mathbf{z}}$ are the coordinate vectors. Thus, the spontaneous emission enhancement factor is $\beta=\beta_{x}+\beta_{y}+\beta_{z}$.

The following section provides an overview on the theory of the FEM for electromagnetics and describes the implementation of the Green's function and LDOS evaluation in the 3D FEM Maxwell solver. Computation results obtained for different cavity configurations will be presented in Sect. 3 where they are compared to characterization results. The last section provides a conclusion and an outlook on further developments.

\section{Computing the LDOS}

The FEM is one approach to solve the frequency domain wave equation

$$
\nabla \times\left(\overline{\bar{\mu}}^{-1} \nabla \times \mathbf{E}\right)-k^{2} \overline{\bar{\epsilon}} \mathbf{E}=-j k Z_{0} \mathbf{J}
$$

for an arbitrary geometry. In this equation is $k=\omega / c$ the wave number, $\mathbf{E}$ the electric field, J the source current density, $\overline{\bar{\mu}}$ the permeability tensor, $\overline{\bar{\epsilon}}$ the dielectricity tensor, and $Z_{0}$ the vacuum wave impedance. The rational behind the FEM is to define a functional whose steady state solutions are the solutions to the underlying partial differential equation problem, in our 
case given in Eq. (5) (Jin 2002). The functional

$$
F(\mathbf{E})=\int_{\Omega} \nabla \times \mathbf{E} \overline{\bar{\mu}}^{-1} \nabla \times \mathbf{E}-k^{2} \mathbf{E} \overline{\bar{\epsilon}} \mathbf{E}+j k Z_{0} \mathbf{E} \mathbf{J} d V
$$

derived for Eq. (5) supports non-Hermitian dielectricity and permeability tensors. Thus, it is feasible to incorporate material losses and absorbing boundary conditions. The volume integration is carried out on the simulation domain $\Omega$ subdivided into a number of tetrahedral elements. On each of these elements a set of base functions is defined. They determine the electric field or the current source density by appropriately associating weights to the base functions within each element. Moreover, these base functions are associated with vertices, edges or faces, so that neighboring elements share the weights. The degrees of freedom (DOFs) are the set of base function weights for a given simulation domain $\Omega$ and are represented by the vector $\psi$.

The choice of the base functions is crucial because of its great influence on the quality of the solution. Solving the wave equation is supported by Nédélec base functions (Nédélec 1986). The 3D FEM Maxwell Solver incorporates a hierarchic element basis (Ainsworth and Coyle 2003). The first order functions of this basis are all associated with the edges. By means of the base functions the functional (6) is subdivided and evaluated over each element yielding a matrix equation. Minimizing this equation yields the linear system of equations

$$
\left(\mathbf{A}-k^{2} \mathbf{B}\right) \boldsymbol{\psi}=-j k Z_{0} \mathbf{C}_{J} \boldsymbol{\phi}
$$

where the symmetric matrices $\mathbf{A}, \mathbf{B}$, and $\mathbf{C}_{J}$ originate from the base function integrals of the functional (6). The source current density vector $\mathbf{J}$ appears in the vector $\boldsymbol{\phi}$. The direct solver Pardiso (Schenk 2000) provides the initial solution of a source sweep for this large but rather sparse linear system of equation. The Krylov space solver GMRes (Saad and Schultz 1986) then computes the subsequent solutions of the source sweep, with the initially computed direct solver acting as preconditioner.

Computing the spatial resolution of the Green's functions for a small frequency range is efficiently supported by the source problem Eq. (5). The definition of the electric dyadic Green's function $\mathcal{G}_{11}$ is

$$
\mathbf{E}(\mathbf{r})=-j k Z_{0} \int_{V} \mathcal{G}_{11}\left(\mathbf{r}, \mathbf{r}^{\prime}\right) \mathbf{J}\left(\mathbf{r}^{\prime}\right) d^{3} r^{\prime}
$$

that immediately yields the wave equation representation

$$
\nabla \times\left(\overline{\bar{\mu}}^{-1} \nabla \times \mathcal{G}_{11}\left(\mathbf{r}, \mathbf{r}^{\prime}\right)\right)-k^{2} \overline{\bar{\epsilon}} \mathcal{G}_{11}\left(\mathbf{r}, \mathbf{r}^{\prime}\right)=\overline{\bar{I}} \delta\left(\mathbf{r}-\mathbf{r}^{\prime}\right)
$$

for this Green's function. The latter equation is viable for the finite element solver because it is a special form of the source problem. The Dirac delta function represents a dipole that is approximated considering the relation

$$
\lim _{V \rightarrow 0} \int_{V} \mathbf{J}\left(\mathbf{r}-\mathbf{r}^{\prime}\right) d^{3} r^{\prime}=\hat{\mathbf{p}} \cdot 1 \mathrm{Am}
$$

for the dipole moment where $\hat{\mathbf{p}}$ is the polarization vector (Jackson 1975). The smallest entity providing a dipole moment is one component of the source vector $\phi$ that is associated with an edge for first order elements as noted before. Higher order elements reuse the first order element base functions so that there is no need to include higher order base functions in the dipole moment evaluation. As the current density vector is determined by the base functions the polarization direction of each source vector component $\phi_{e}$ is fixed. This problem can be 

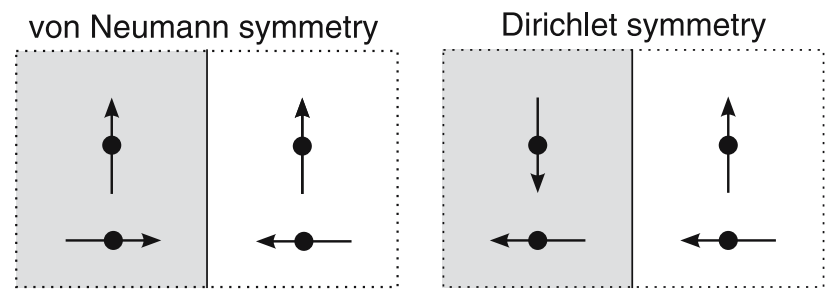

Fig. 2 Dipoles mirrored by a von Neumann and Dirichlet boundary condition. The white area contains the original dipoles and the gray area the mirrored ones. A superposition of both mirroring schemes will cancel the mirrored dipoles

solved by placing the dipole on a vertex $v$ of the grid. Consequently, the linear combination of all dipole moments associated with the edges connected to that vertex yields the total dipole moment

$$
\mathbf{p}_{v}=\sum_{e: v \in e} \phi_{e} \mathbf{p}_{e}
$$

where $\mathbf{p}_{e}$ is the dipole moment associated with one edge. The latter corresponds to the contribution of all elements $s$ sharing the edge $e$ :

$$
\mathbf{p}_{e}=\sum_{s: e \in s} \int_{s} \mathbf{N}_{s, e} d V,
$$

where the $\mathbf{N}_{s, e}$ are the base functions of the element $s$ associated with the edge $e$. As the base functions solely depend on the element geometry the integral (12) and thus the dipole moment associated with one edge are constant for a given geometry. At least 3 but usually more than 3 edges connect to a vertex. Therefore Eq. (11) generally yields an underdetermined system of equations that is suggestive to be solved applying a minimum norm for $\boldsymbol{\phi}$. Changing the location of the dipole affects only the source vector $\phi$. Once an $L D L^{T}$ decomposition (Schenk 2000; Golub and van Loan 1989) of $\mathbf{A}-k^{2} \mathbf{B}$ has been obtained it allows the computation of the solution vector $\psi$ by a forward-backward substitution. This is equivalent to a matrix multiplication with $L^{-T} D^{-1} L^{-1}$ and hence the latter is equivalent to a discrete version of the Green's function.

Another way to obtain the Green's function is the eigenfunction expansion based on all eigenmodes (Tai 1994). This method is not applied here because it is hard to estimate the error if the number of contributing eigenmodes is limited to a reasonably small value. Note that the full eigenfunction expansion is nothing less than an eigentransformation of the matrix $\mathbf{A}-k^{2} \mathbf{B}$.

Some attention has to be paid to symmetry boundaries in the model. As noted previously the FE solver exploits symmetries in the model in order to enhance performance. Any symmetry boundary implies a mirror dipole for the one on the right side of Eq. (9) thus tampering the Green's function. Considering the mirroring properties of a von Neumann type boundary condition $\left(E_{n}=0\right)$ and of a Dirichlet type boundary condition $\left(E_{t}=0\right)$, as illustrated in Fig. 2, the total number of arrangements of mirrored dipoles amounts to $2^{n}$ where $n$ is the number of symmetry boundaries. Hence, the effect of the mirror dipoles will be cancelled by a superposition of the computational results of all possible combinations of von Neumann and Dirichlet conditions for each symmetry boundary.

Due to symmetry the simulation space of the investigated photonic crystal cavities covers only one eighth of the total cavity. A dipole placed at the center of the cavity, i.e., at the intersection of all three symmetry planes, and oriented along one principal axis permits only 
one combination of boundary conditions. These are always Dirichlet on the symmetry plane perpendicular to the orientation and von Neumann on the remaining symmetry planes as can be deduced from the symmetry schemes shown in Fig. 2. On the contrary, all other combinations of symmetry boundary conditions force the dipole to cancel itself. Thus the computation of the Green dyad at the center of the cavity confines to 3 different combinations of boundary conditions. A dipole that is not located on any boundary requires all $2^{3}$ combinations to be considered.

\section{Modes and LDOS in photonic crystal cavities}

In the subsequent pararaph we are investigating photonic crystal cavities in a $320 \mathrm{~nm}$ thick GaAs membrane. The hexagonal photonic crystal has a lattice constant of $a=340 \mathrm{~nm}$ and a filling factor of $35 \%$ for operation around $1300 \mathrm{~nm}$. The cavity is formed by defect holes yielding the $\mathrm{H} 1$ and L3 cavity types depicted in Fig. 1. Unlike shown in the schematic the cavity is surrounded by 8 consecutive rows of holes in each principal direction. We assume the emitter to be located in the center of the cavity.

The fabrication of these structures and the optical characterization by microphotoluminescence are described in (Francardi et al. 2006). In this experiment the emitters are realized growing a layer of self-assembled InAs/GaAs quantum dots (QDs) with a wide emission spectrum around $1300 \mathrm{~nm}$ (Fiore et al. 2001). Due to the limited emission bandwidth of the embedded quantum dots samples with a different scaling of the hole distance have been characterized thus sweeping the emission spectrum with respect to the normalized wave number $a / \lambda=a k /(2 \pi)$.

The structures are aimed for highly efficient single photon extraction from a single quantum dot. This requires designing the optical environment such that the quantum dot efficiently couples to a single cavity mode to reduce the spontaneous emission lifetime and to increase the coherence time to the photon lifetime of the cavity simultaneously. Therefore the investigation concentrates particularly on those cavities providing a small effective volume. These are the H1 cavity with one defect hole and the L3 cavity with 3 defect holes in line. The latter one has been found to provide comparatively high quality factors (Akahane et al. 2003).

The numerical analysis of the LDOS has been performed in two ways. On the one hand a frequency sweep of the LDOS at the cavity center, the intersection of all three symmetry planes, has been performed and plotted for each polarization direction. On the other hand the spatial dependency of the LDOS in the vicinity of the cavity has been investigated at particular frequencies. The first experiment allows to obtain a measure for the spontaneous emission enhancement one can expect and therefore directly compares to the PL characterization. The second experiment enables the fully coupled electro-opto-thermal simulation by providing the local spontaneous emission enhancement which allows for the computation of the local spontaneous emission rate.

For the sake of clarity the relative LDOS $\beta$ is shown in the figures rather than the LDOS $\rho_{e}$. The LDOS value in bulk GaAs is governed by the refractive index. Hence, a value of $\beta$ below 3.42 which is the average refractive index of GaAs in the observed frequency range represents spontaneous emission suppression while a value above 3.42 represents enhancement. Note that the refractive index dispersion and anisotropy have not been considered in the simulations so far. The normalized photoluminescence (PL) indicated in the frequency sweep figures has been obtained by filtering the PL curve to remove the resonant spontaneous emission, and dividing the original curve by the filtered one. Though this is a rather heuristic procedure the background PL allows for a approximate reconstruction of the PL curve without the cavity. 
Table 1 Modes of the $\mathrm{H} 1$ and L3 cavity

$k$ is the wave number and $Q$ the quality factor. The polarization applies at the cavity center. z-polarized modes have been omitted

\begin{tabular}{llllll}
\hline H1 & $k[1 / \mathrm{m}]$ & $Q$ & L3 & $k[1 / \mathrm{m}]$ & $Q$ \\
\hline X1 & $5.0579 \cdot 10^{6}$ & 176 & X1 & $4.6303 \cdot 10^{6}$ & 4654 \\
Y1 & $5.0548 \cdot 10^{6}$ & 176 & Y1 & $5.0102 \cdot 10^{6}$ & 520 \\
& & & Y2 & $5.0512 \cdot 10^{6}$ & 135 \\
& & & X2 & $5.5617 \cdot 10^{6}$ & 345 \\
\hline
\end{tabular}

The spatial distribution of the LDOS indicates the preferred polarization direction for each vertex by the vector

$$
\hat{\mathbf{p}}_{\beta}=\frac{\left(\beta_{x}, \beta_{y}, \beta_{z}\right)^{T}}{\left|\left(\beta_{x}, \beta_{y}, \beta_{z}\right)\right|} .
$$

If this vector is oriented parallel to a principal axis, there is a preferred polarization in the direction of this axis. However, if it is parallel to the $(1,1,1)^{T}$ vector there is no preference in the polarization at all rendering the LDOS isotropic. Note that the LDOS polarization vector is not subject to boundary constraints. The investigated eigenmodes show a strong preference for the $\mathrm{x}$ - or $\mathrm{y}$-polarization in the cavity. The $\mathrm{z}$-polarization LDOS is spatially more or less constant. Hence, it is omitted in the spatial plots which indicate only the value $\beta_{x y}=\beta_{x}+\beta_{y}$.

The $\mathrm{H} 1$ cavity provides a strong confinement in $\mathrm{x}$ - and $\mathrm{y}$ - direction and therefore substantial vertical leakage yielding eigenmodes with a relatively small quality factor as indicated in Table 1. The hexagonal lattice provides an almost isotropic confinement of the optical mode and thus the $\mathrm{x}$-polarized mode $\mathrm{X} 1$ and the y-polarized mode $\mathrm{Y} 1$ are quasi degenerate. ${ }^{1}$ Due to their strong overlap there is practically no way to tell both polarization modes apart which is confirmed by the LDOS frequency sweep and the PL characterization shown in Fig. 3. The variation of the z-polarization LDOS is not reflected in the characterization because the z-polarized emission is largely suppressed in self-assembled quantum dots (Wang et al. 1999).

Despite the small Q-factor $Q=176$ of the $\mathrm{x}$ - and y-polarization modes, the H1 cavity provides a substantial spontaneous emission enhancement factor $\beta_{\max , H 1}=96$ in its center as shown in Fig. 4. This enhancement is due to the tight confinement of the mode and the colocated contribution of the $\mathrm{x}$ - and the y-polarization mode. The spatial distribution of the LDOS depicted in Fig. 7 is almost direction independent and shows that the LDOS attains its maximum in the center where is is isotropic with respect to the $\mathrm{x}$ - and $\mathrm{y}$-polarization.

The $\mathrm{H} 1$ cavity shows a strong spontaneous emission suppression at $k=5.32 \cdot 10^{6} \mathrm{~m}^{-1}$ which affects the $\mathrm{x}$ - as well as the $\mathrm{y}$-polarization due to the isotropy. The electric field is phase-shifted by 90 degrees with respect to the dipole source current. Thus the power $P_{\text {rad }}=\int \mathbf{E} \mathbf{J} d V$ becomes imaginary yielding idle power and the dipole cannot radiate. Figure 8 shows that this effect confines to a very small area of about $0.01 \mu \mathrm{m}^{2}$ in the center of the cavity. This area is surrounded by a local maximum of the $\mathrm{x}$ - and y-polarization LDOS. As the PL characterization reflects the integral emission of all QDs located in the suppression region and its vicinity the spontaneous emission suppression is not reflected in the PL spectra.

The L3 cavity provides an anisotropic confinement of the mode. This property is reflected in the eigenmodes for $\mathrm{x}$ - and $\mathrm{y}$-polarization presented in Table 1 . Not only the resonant

1 The modes X1 and Y1 are not mathematically degenerate. However, in a physical interpretation of degeneracy the frequency spacing of the modes is much smaller than the linewidth so that they cannot be discriminated. 


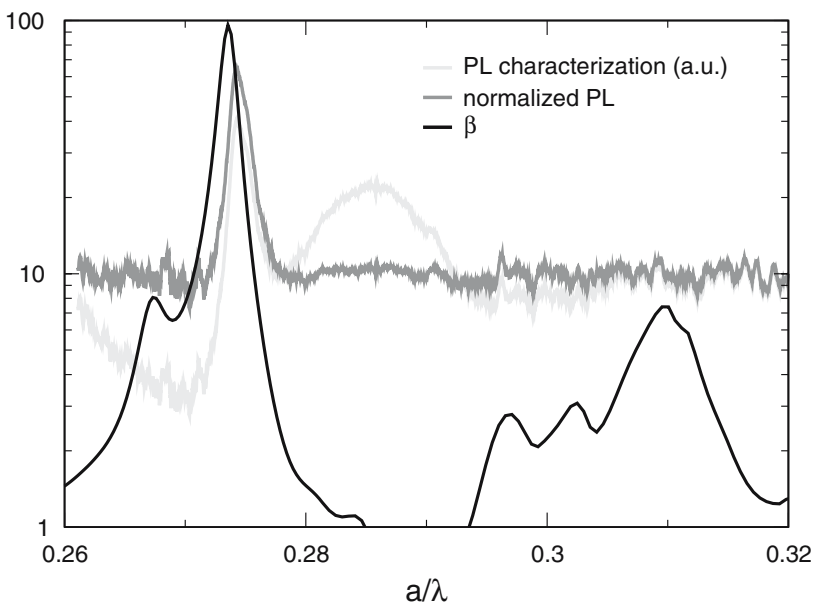

Fig. 3 Relative LDOS of the H1 cavity at the center compared to the PL characterization of the H1 cavity

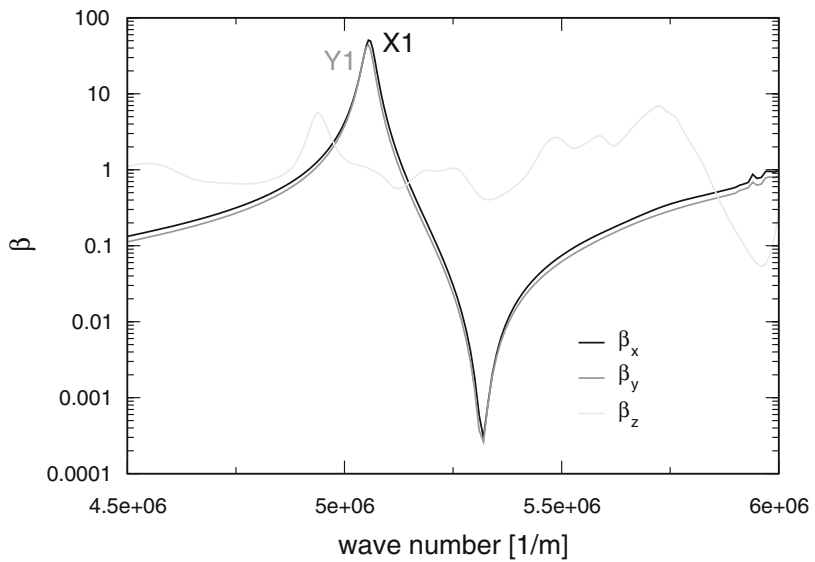

Fig. 4 Relative LDOS of the H1 cavity at the center for each diagonal component of the Green dyad

frequencies differ, but there is also a strong difference in the quality factors. Due to its high quality factor of $Q=4654$ the mode $\mathrm{X} 1$ provides a high spontaneous emission enhancement as indicated by Fig. 5. There is however a strong polarization dependence so that the $\mathrm{X}$ - and the y-polarization modes do not coincide.

Figure 6 shows the relative LDOS with the PL characterization clearly reflecting these modes. There is a shift of the X1 mode towards a higher frequency and a shift of the X2 mode towards a lower frequency with respect to the simulation. This shift is due to the aforementioned refractive index variation of bulk GaAs between 3.41 and 3.44 in the observed frequency range. Even though only the spontaneous emission enhancement in the center has been computed and not the spatial average the simulation results agree quite well with the normalized PL considering the linewidth and the relative height of the modes. There is however a difference in the X1 mode because the measured quality factor does not reach the theoretical one due to fabrication tolerances like non-vertical hole walls, surface roughness, and imperfect hole periodicity. 


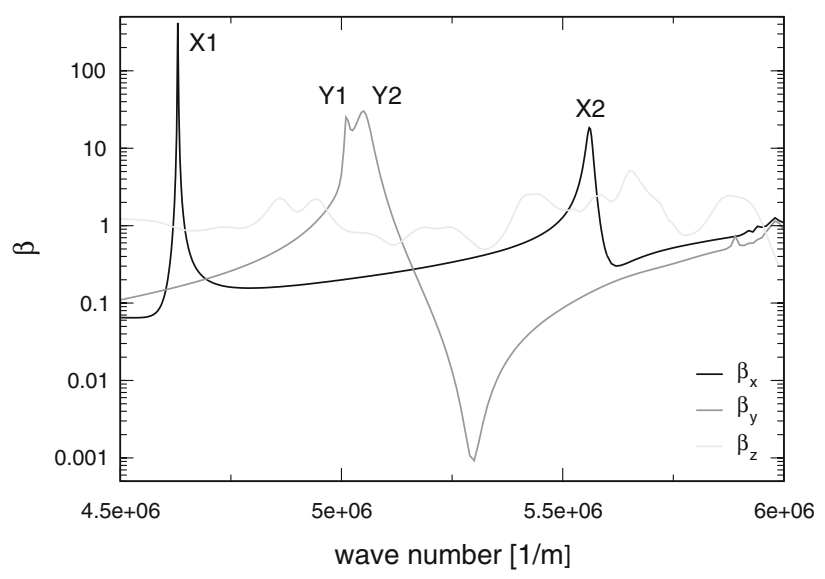

Fig. 5 Relative LDOS of the L3 cavity at the center for each diagonal component of the Green dyad

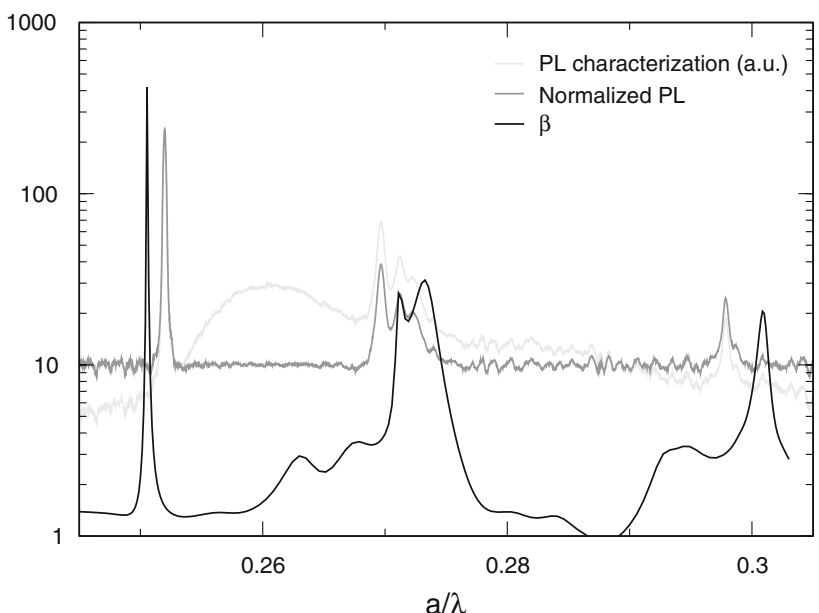

Fig. 6 Relative LDOS of the L3 cavity at the center compared to the PL characterization of the L3 cavity

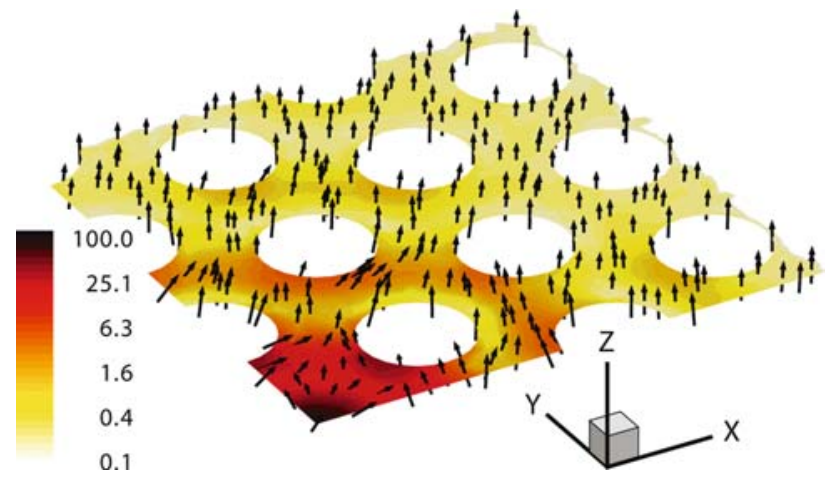

Fig. 7 Spatial LDOS variation $\beta_{x y}$ in the $\mathrm{x}-\mathrm{y}-$ plane of the $\mathrm{H} 1$ cavity computed at the resonant frequency of mode $\mathrm{X} 1$. The LDOS is almost isotropic in the center of the cavity 


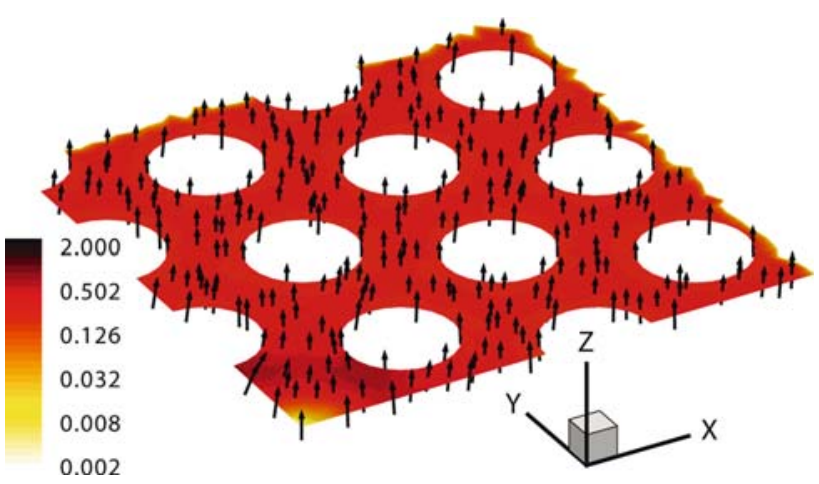

Fig. 8 Spatial LDOS variation $\beta_{x y}$ in the $\mathrm{x}-\mathrm{y}$-plane of the $\mathrm{H} 1$ cavity at the suppression point $k=5.32 \cdot 10^{6} \mathrm{~m}^{-1}$. The suppression space is quite small so that this effect cannot be observed in the characterization

Figure 5 shows that $\beta_{y}$ exhibits a dip at $k=5.30 \cdot 10^{6} \mathrm{~m}^{-1}$ that is almost collocated with the dip observed for $\mathrm{X}$ - and y-polarization within the H1 cavity. Also the Y1 mode of the $\mathrm{H} 1$ and the Y2 mode of the L3 cavity almost coincide. The collocated suppression and mode for the $y$-polarization indicate that the confinement is strongly influenced by the holes confining the $\mathrm{H} 1$ cavity in $\mathrm{x}$-direction. The holes confining the $\mathrm{H} 1$ cavity in $\mathrm{y}$-direction are less important though there is a small decrease of the quality factor in the L3 cavity. The small wobble in the y- and x-polarization LDOS that appears at high frequencies in Figs. 5 and 4 is due to the sweep leaving the photonic band gap.

Notably the peak LDOS of the H1 cavity is only by a factor of $\beta_{\max , L 3} / \beta_{\max , H 1}=4.35$ lower than the one of the L3 cavity despite the ratio of the quality factors of the fundamental modes $\left(Q_{L 3, X 1} / Q_{H 1, X 1}=26.5\right)$. By means of the approximate relation for the spontaneous emission enhancement (Meschede 1992) the effective modal volume may be estimated to $V_{e f f}=6 \pi Q / \beta_{\max } / k^{3} \hat{\mathbf{e}} \hat{\mathbf{p}}$. Considering the colocated contribution of the $\mathrm{X} 1$ and the $\mathrm{Y} 1$ mode the effective cavity volume ratio evaluates to $V_{e f f, L 3} / V_{e f f, H 1}=3.96$. The result confirms the influence of the effective volume on the spontaneous emission enhancement.

Figure 9 shows the spatial variation of the LDOS at the frequency of mode X1 for the L3 cavity. The spatial distribution resembles the X1 eigenmode due to its dominance and has its peak at the center $\left(\beta_{\max , L 3}=417.5\right)$. There is a strong preference for the $\mathrm{x}$ - and $\mathrm{y}$-polarization which coincides with the polarization preference of the quantum dots. At some distance from the cavity center the z-polarization becomes dominant resulting in a suppression of the spontaneous emission.

The spatial maximum of the LDOS does not stringently coincide with the cavity center. Figure 10 shows the LDOS at the resonance of mode Y1 clearly indicating that the LDOS maximum coincides with the outer defect holes of the L3 cavity yielding two identical global maxima. This arrangement enables the efficient coupling of two quantum dots if each one is placed in one LDOS maximum. Apart from the location the LDOS distribution and polarization preference is quite similar to the one of the $\mathrm{H} 1$ cavity at the $\mathrm{X} 1 / \mathrm{Y} 1$ mode.

\section{Conclusion and outlook}

The design of photonic crystal cavities serving as microcavities for efficient incoherent emitters requires highly sophisticated simulation tools to predict the spatial and frequency dependence of the spontaneous emission enhancement. While the approximate equation 


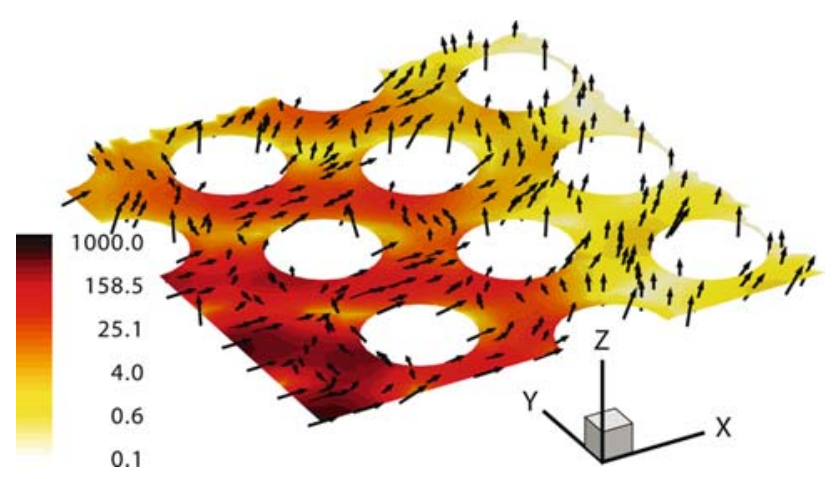

Fig. 9 Spatial LDOS variation $\beta_{x y}$ in the $x-y-$ plane of the L3 cavity computed at the resonant frequency of mode X1. This mode provides not only the highest peak LDOS but also the largest penetration

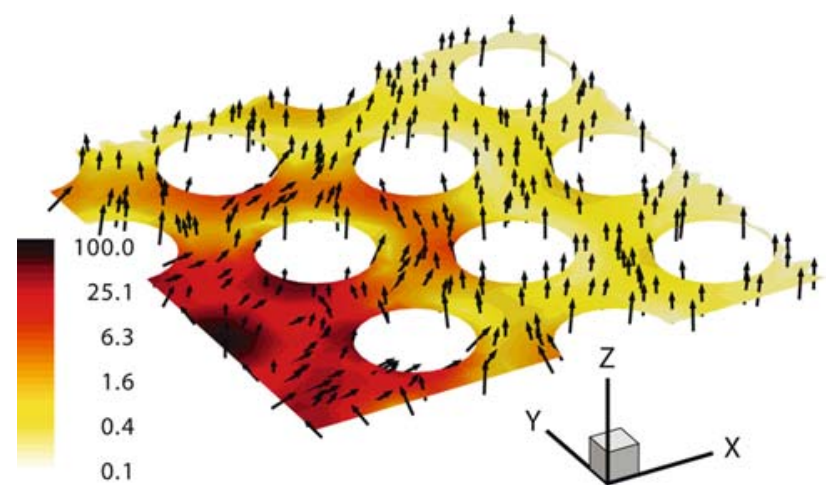

Fig. 10 Spatial LDOS variation $\beta_{x y}$ in the $x-y-p l a n e$ of the L3 cavity computed at the resonant frequency of mode Y1. The LDOS maximum does not coincide with the cavity center here

(Meschede 1992) might predict correct values for some modes it might fail for other ones. The 3D Finite Element Maxwell solver presented in this work enables the computation of the spatial LDOS picture and its frequency dependence for any type of cavity while the approximate equation provides only an estimate for the spatial field maximum if the mode separation is high enough. Moreover, the 3D FEM Maxwell solver provides optimized performance for fully coupled electro-opto-thermal simulations. The fully coupled simulation will enable the detailed optimization of photonic crystal light emitters. Though not yet calibrated, the simulated LDOS shows very good agreement with the measured data confirming the accuracy and performance of the 3D FEM Maxwell solver.

First results obtained for the $\mathrm{H} 1$ and L3 photonic crystal cavities show that there is potential for optimizing the spontaneous emission enhancement. The H1 cavity is very promising mainly due to its small effective volume providing a high spontaneous emission enhancement. However, further optimization will not only be carried out for the spontaneous emission enhancement. Another important feature to be investigated in future is the coupling of the emission to an optical system.

Acknowledgements We thank A. Fiore, L. Balet from the Ecole Polytechnique Fédérale de Lausanne and M. Francardi, A. Gerardino from the CNR Institue of Photonics and Nanotechnology, for providing the experimental data, valuable discussions, and reviewing this manuscript. Funding by the ESF under the COST P11 project C05.70 is gratefully acknowledged. 


\section{References}

Ainsworth, M., Coyle, J.: Hierarchic finite element bases on unstructured tetrahedral meshes. Int. J. Numer. Meth. Eng. 58, 2103-2130 (2003)

Akahane, Y., Asano, T., Song, B.-S., Noda, S.: High-Q photonic nanocavity in a two-dimensional photonic crystal. Nature 425, 944-947 (2003)

Alivisatos, P.: The use of nanochrystals in biological detection. Nat. Biotechnol. 22, 47-52 (2004)

Berenger, J.-P.: A perfectly matched layer for the absorption of electromagnetic waves. J. Comput. Phys. 114, 185-200 (1995)

Coldren, L.A., Corzine, S.W.: Diode Lasers and Photonic Integrated Circuits. John Wiley (1995)

Fiore, A., Oesterle, U., Stanley, R.P., Houdré, R., Lelarge, F., Ilegems, M., Borri, P., Langbein, W., Birkedal, D., Hvam, J.M., Cantoni, M., Bobard, F.: Structural and Electrooptical Characteristics of Quantum Dots Emitting at $1.3 \mu \mathrm{m}$ on Gallium Arsenide. IEEE J. Quantum Elect. 37, 1050-1058 (2001)

Francardi, M., Balet, L., Gerardino, A., Monat, C., Zinoni, C., Li, L.H., Alloing, B., Thomas, N.L., Houdré, R., Fiore, A.: Quantum dot photonic crystal nanocavities at $1300 \mathrm{~nm}$ for telecom-wavelength single-photon source. Phys. Status Sol. (C) 3, 3693-3696 (2006)

Golub, G.H., van Loan C.F.: Matrix Computations. The John Hopkins University Press (1989)

Henkel, C., Sandoghdar, V.: Single-molecule spectroscopy near structured dielectrics. Opt. Commun. 158, 250-262 (1998)

Imamoglu, A.: Are quantum dots useful for quantum computation?. Physica E 16, 47-50 (2003)

Jackson, J.: Classical Electrodynamics. John Wiley (1975)

Jin J.: The Finite Element Method in Electromagnetics, 2nd edn. John Wiley (2002)

Joulain, K., Carminati, R., Mulet, J.-P., Greffet, J.-J.: Definition and measurement of the local density of electromagnetic states close to an interface. Phys. Rev. B 68, 245405 (2003)

Meschede, D.: Radiating atoms in confined space: from spontaneous emission to micromasers. Phys. Rep. 211, 201-250 (1992)

Nédélec, J.C.: A New Family of Mixed Finite Elements in $\mathrm{IR}^{3}$, . Numer. Math. 50, 57-81 (1986)

Purcell, E.M., Torrey, H.C., Pound, R.V.: Resonance absorption by nuclear magnetic moments in a solid. Phys. Rev. 69, 37-38 (1946)

Saad, Y., Schultz, M.H.: GMRES: A Generalized Minimal Residual Algorithm for Solving Nonsymmetric Linear Systems. SIAM J. Sci. Stat. Comput. 7, 856-869 (1986)

Schenk, O.: Scalable Parallel Sparse LU Factorization Methods on Shared Memory Multiprocessors. PhD thesis No. 13515, ETH Zürich. (2000)

Song, B.-S., Noda, S., Asano, T., Akahane, Y.: Ultra-high-Q photonic double heterostructure nanocavity. Nat. Mat. 4, 207-210 (2005)

Streiff, M., Witzig, A., Pfeiffer, M., Royo, P., Fichtner, W.: A Comprehensive VCSEL Device Simulator. IEEE J. Sel. Top. Quant. 9, 879-891 (2003)

Streubel, K., Linder, N., Wirth, R., Jaeger, A.: High brightness AlGaInP light-emitting diodes. IEEE J. Sel. Top. Quant. 8, 312-332 (2002)

Tai, C.T.: Dyadic Green Functions in Electromagnetic Theory. IEEE Press (1994)

Teixeira, F.L., Chew, W.C.: Complex space approach to perfectly matched layers: a review and some new developments. Int. J. Numer. Model. 13, 441-455 (2000)

Vučković, J., Lončar, M., Mabuchi, H., Scherer, A.: Optimization of the Q factor in photonic crystal microcavities. IEEE J. Quantum Elect. 38, 850-856 (2002)

Wang, L.-W., Kim, J., Zunger, A.: Electronic structures of [110]-faceted self-assembled pyramidal InAs/GaAs quantum dots. Phys. Rev. B 59, 5678-5687 (1999)

Witzigmann, B., Witzig, A., Fichtner, W.: A multidimensional laser simulator for edge-emitters including quantum carrier capture. IEEE T. Electron Dev. 47, 1926-1934 (2000) 